\section{Synchrotron Light to Explore Matter. IMEDIASoft/Springer-Verlag/ESRF, 2000. CD-ROM. Price DM 98. ISBN 3540148884.}

This is a quite exceptional introduction to the world of X-ray synchrotron radiation research and technology. Unlike a book, the CD-ROM adds new opportunities such as interactive schematic diagrams and video clips which enhance both understanding and readability. Multiple entry points allow easy exploration and concentration on the reader's own interests, while the two- and threedimensional animations and diagrams considerably aid understanding and learning. The level and style are clearly aimed at a general audience which needs to be informed.

Following a brief history of the development of synchrotron radiation research, the structure of the CD-ROM is described: a twodimensional CD map is available at all times covering four subject areas, i.e. light and matter, technology, methods and applications. Each topic is preceded by a well composed topical video introduction which is easily avoided after first viewing by navigating from the $\mathrm{CD}$ map.

Light and Matter gives an introduction to the nature of light, properties of light, X-rays and synchrotron light in about 46 pages and summarizes, in similar detail, descriptions of atoms, interatomic bonds, order and disorder, and properties of matter.

Technology is a larger section covering generalities (electrons, Lorentz force, energy scales etc.), injection, storage ring (components, insertion devices, operation modes etc.), general features (hutches etc.), (X-ray) optics, sample (including diffractometers, sample environment etc.), detectors and control/data acquisition and safety.

Methods, within the style set provides a comprehensive survey, about 50 pages, of what can be performed with synchrotron X-rays: imaging, absorption and spectroscopy, scattering, and diffraction. Complementary methods, such as neutrons, electrons, nuclear magnetic resonance and mass spectroscopy, are only very briefly described.

Applications abound! The science and technology clips form the major part of the CD-ROM, almost 300 pages, far more than would be possible in a book and far easier to browse, appreciate and study in detail. The classification of entries as physics (surfaces, magnetism, extreme conditions, various examples), materials, biology, medicine, chemistry and environment is necessarily rather arbitrary, but the CD map is always present and makes navigation easy. To the uninitiated new reader, the scope of activities will be awe-inspiring, including (as a very small sample): solid-liquid interfaces, magnetic domains, ice, quasicrystals, fullerines, bottles made of PET, concrete, spider silk, chocolate, amino acids, DNA, blue tongue virus, hair, angiography, Alzheimer's disease, osteoporosis, fuel cells, surfactants, make-up in ancient Egypt, solar energy, decontaminating plants, enzymes against the greenhouse effect, catalytic converters and chlorine bleach.

Throughout, the style follows closely that of the established ESRF Highlights series of publications, though with far fewer literature references and suggestions for further reading. Overall there are very few equations, just Bragg's Law and $E=h v$, but not Snell's Law, the Lorentz force, nor a definition of the characteristic energy of synchrotron light $E_{c}$.

I will conclude with a description of some of the presentational highlights which bring the subject to life in a way which simply cannot be achieved in book form.
In the sections describing the Technology of synchrotron radiation facilities, the reader can merge to and from an actual photograph of hardware, e.g. an ESRF beamline, to a labelled block diagram of the key functional parts. The nuts, bolts and brackets are stripped away exposing the key features clearly and immediately. Animations allow one to see the motions and operational surroundings of whole beamlines, experimental enclosures, apparatus and sample environment at many different levels of detail. In the same way, there are excellent descriptions of how the storage ring is injected and operated, how the radiation emerges from the ring, and how insertion devices work. The animation showing how the spectrum from an undulator changes as the gap is altered is particularly well executed.

In the Science sections, three dozen or so atomic models are available which can be rotated in two or three dimensions on command, something which is commonplace to crystallographers but which is almost never available to scientists and engineers who are outside that field, but who might become users. Molecules (RasMol files) from ammonia, through buckyballs, to DNA are in the database; one of my favourites is a three-dimensional zeolite model which one can rotate to show very easily the various symmetry directions and the huge 'holes' in the structure which dictate the catalytic function.

In the Medical sections, the reader can follow a beating heart, section a brain, watch cell division or see the results of microbeam radiation therapy. In the Materials section, there is a very nice animation of the LIGA masking process used in the manufacture of three-dimensional structures in silicon. One could continue to enthuse across the whole of science and technology, but that is for the readers who want to know more about the scope of research with Xrays from synchrotron radiation sources to do. You must buy your own copy!

Thoughtfully, the CD-ROM includes a list of all the world facilities and their contact particulars, a very nice world map and a list of some industrial users and vendors, though this seems to be very specific to the ESRF and the sponsors of the CD-ROM production.

Finally, there are a few problems which are inevitable with a work of this range and scope.

Seriously, one piece of the physics is wrong. The treatment of refraction shows animations which allow the reader to change the angle of incidence of light (X-rays) on a plane boundary between media. The ray refracted into the medium is constructed with the lower medium as denser than the upper whereas the totally reflected rays at grazing incidence are in the upper medium. The error results in a very strange jump in the direction of the refracted ray in the denser medium at the transition to total reflection.

The quality of error detection in both spelling and technical matters, such as the linkages between topics and pages, is very high.

At any time the reader can switch text, links and speech between French and English - a clear sign for future development and an invitation for the second edition to incorporate several more languages. An outline CD-ROM was distributed free with Synchrotron Radiation News a few months ago; a major impact would be achieved if the full version could be made available on the same basis.

\section{Michael Hart}

HH Wills Physics Laboratory

Bristol University

Tyndall Avenue

Bristol BS8 1TL

UK 